\title{
Otras disposiciones de interés en la Comunidad Autónoma
}

\begin{abstract}
Decreto 140/2013, de 1 de octubre, por el que se establece la estructura orgánica de la consejería de Igualdad, Salud y Políticas Sociales y del Servicio Andaluz de Salud. Decreto 141/2013, de 1 de octubre, por el que se establece la estructura orgánica de la consejería de Agricultura, Pesca y Desarrollo rural. Decreto 142/2013, de 1 de octubre, por el que se establece la estructura orgánica de la consejería de Medio Ambiente y ordenación del territorio. Boletín Oficial de la Junta de Andalucía 2 de octubre 2013.
\end{abstract}

La Ley 9/2007, de 22 de octubre, de la Administración de la Junta de Andalucía, regula en el título II la organización de la Administración de la Junta de Andalucía, así como el régimen general de los órganos y unidades administrativas, definiendo su estructura central y territorial .Por otra parte el Decreto de la Presidenta 4/2013, de 9 de septiembre, de la Vicepresidencia y sobre reestructuración de consejerías, ha producido una remodelación de la administración autonómica que ahora se completa con estos tres decreto.

Decreto 163/2013, de 8 de octubre, por el que se modifica el Decreto 342/2012, de 31 de julio, por el que se regula la organización territorial provincial de la Administración de la Junta de Andalucía. Boletín Oficial de la Junta de Andalucía, 199, de 9 de octubre de 2013.

Con este decreto se pretende adaptar a lo dispuesto en el Decreto de la Presidenta 4/2013, de 9 de septiembre, de la Vicepresidencia y sobre reestructuración de consejerías, los órganos directivos periféricos de la Administración de la Junta de Andalucía, manteniendo las Delegaciones del Gobierno de la Junta de Andalucía y cinco Delegaciones. Así, las Delegaciones del Gobierno de la Junta de Andalucía, primera autoridad de la Administración de la Comunidad Autónoma de Andalucía en la provincia, continúan integrando los servicios periféricos de las consejerías de Administración Local y relaciones Institucionales, de Hacienda y Administración Pública y de Justicia e Interior. Por otra parte, de las cinco Delegaciones territoriales de la Junta de Andalucía, tres desarrollarán en la en la provincia competencias de una sola consejería, dado el importante volumen competencial que dichas consejerías han asumido mediante el Decreto de la Presidenta 4/2013, de 9 de septiembre. Son las Delegaciones territoriales de economía, Innovación, ciencia y empleo, de Igualdad, Salud y Políticas Sociales y de 
educación, cultura y Deporte. Las otras dos Delegaciones territoriales de la Junta de Andalucía, en aras a lograr la máxima eficiencia en la gestión de los recursos públicos y persiguiendo el acercamiento real de la Administración a la ciudadanía, integrarán los servicios periféricos de dos consejerías. Estas Delegaciones territoriales de la Junta de Andalucía son las de Fomento, Vivienda, turismo y comercio y la de Agricultura, Pesca y Medio Ambiente.

\footnotetext{
Acuerdo de 3 de diciembre de 2013, del consejo de Gobierno, por el que se aprueba la estrategia de infraestructuras de telecomunicaciones de Andalucía 2020. Boletín Oficial de la Junta de Andalucía 226 de 16 de diciembre de 2013.
}

La sociedad está viviendo un momento de transformación caracterizado por el impacto de la crisis, las debilidades estructurales y la intensificación de los retos mundiales. El resultado de este proceso debe constituir la puerta de entrada a una economía distinta, basada en el conocimiento y la innovación, que haga un uso más eficaz de los recursos y con altos niveles de empleo, cohesión social y territorial. Consciente de este reto, la Unión Europea (UE) ha definido la Estrategia Europa 2020 para convertir Europa en una economía cimentada en un crecimiento inteligente, sostenible e integrador. Un crecimiento inteligente implica la consolidación del conocimiento y la innovación como motores del crecimiento futuro.

La Agenda digital para Europa expresa la necesidad de tomar medidas para garantizar el despliegue y la adopción de la banda ancha para todos, a velocidades crecientes, a través de tecnologías tanto fijas como inalámbricas, así como para facilitar la inversión en las nuevas redes ultrarrápidas de Internet abiertas y competitivas que constituirán las arterias de la economía del futuro. Resulta necesario crear los incentivos adecuados para fomentar la inversión privada, complementada por inversiones públicas con objetivos cuidadosamente seleccionados sin que se produzca una nueva monopolización de las redes, así como mejorar la atribución del espectro. 